\title{
Overexpression of plum auxin receptor PsITIR1 in tomato alters plant growth, fruit development and fruit shelf-life characteristics
}

\author{
I. El-Sharkawy ${ }^{1,2}$, S. Sherif ${ }^{1,2}$, W. El Kayal ${ }^{1}$, B. Jones ${ }^{3}$, Z. Lii , A. J. Sullivan ${ }^{5}$ and Subramanian Jayasankar ${ }^{1 *}$
}

\begin{abstract}
Background: TIR1-like proteins are F-box auxin receptors. Auxin binding to the F-box receptor proteins promotes the formation of SCF ${ }^{\text {TRT }}$ ubiquitin ligase complex that targets the auxin repressors, Aux/IAAs, for degradation via the ubiquitin/26S proteasome pathway. The release of auxin response factors (ARFs) from their Aux/IAA partners allows ARFs to mediate auxin-responsive changes in downstream gene transcription. In an attempt to understand the potential role of auxin during fruit development, a plum auxin receptor, PSITIRT, has previously been characterized at the cellular, biochemical and molecular levels, but the biological significance of this protein is still lacking. In the present study, tomato (Solanum lycopersicum) was used as a model to investigate the phenotypic and molecular changes associated with the overexpression of PSITIRT.
\end{abstract}

Results: The findings of the present study highlighted the critical role of PsITIR1 as positive regulator of auxinsignalling in coordinating the development of leaves and fruits. This was manifested by the entire leaf morphology of transgenic tomato plants compared to the wild-type compound leaf patterning. Moreover, transgenic plants produced parthenocarpic fruits, a characteristic property of auxin hypersensitivity. The autocatalytic ethylene production associated with the ripening of climacteric fruits was not significantly altered in transgenic tomato fruits. Nevertheless, the fruit shelf-life characteristics were affected by transgene presence, mainly through enhancing fruit softening rate. The short shelf-life of transgenic tomatoes was associated with dramatic upregulation of several genes encoding proteins involved in cell-wall degradation, which determine fruit softening and subsequent fruit shelf-life.

Conclusions: The present study sheds light into the involvement of PsITIR1 in regulating leaf morphology, fruit development and fruit softening-associated ripening, but not autocatalytic ethylene production. The results demonstrate that auxin accelerates fruit softening independently of ethylene action and this is probably mediated through the upregulation of many cell-wall metabolism genes.

Keywords: Auxin receptors, Auxin/ethylene cross-talk, Cell-wall metabolism, Fruit-set, Fruit firmness, Plant development, Reproductive growth, Shelf-life

\footnotetext{
* Correspondence: jsubrama@uoguelph.ca

${ }^{1}$ Department of Plant Agriculture, University of Guelph, Vineland Station, ON,

Canada

Full list of author information is available at the end of the article
} 


\section{Background}

The phytohormone auxin controls almost every aspect of plant growth and development. At cellular level, auxin regulates cell division, expansion and differentiation [1]. Some short-term effects may reflect direct auxin impact on cell membrane proteins; however, most other responses appear to be due to changes in the transcription of target auxin-responsive genes either by activation or repression $[2,3]$. At the whole plant level, auxin controls essential processes such as apical dominance, lateral root formation, tropic responses, vascular initiation and differentiation, embryogenesis, and fruit development $[4,5]$.

Fruit development is a multiphase process that requires a tight coordination among molecular, biochemical and structural elements. The series of modifications that make the fruit proceed through consequent developmental stages involve many distinctive metabolic pathways. The availability of plant mutants with fruits unable to ripen autonomously has helped us to understand the mechanisms underlying fruit development process in which phytohormones are placed as master regulators, leading to efficient reproductive growth [6, 7]. Among various phytohormones, auxin has received a lot of attention due to its prominent role in controlling widerange of events during plant life, particularly those involved in flower and fruit development $[1,8]$. In flowering plants, auxin is required for floral meristem formation and acts with homeotic genes in determining floral organogenesis [9]. Auxin bioassays highlight the pivotal role played by auxin in regulating the reproductive growth and the final fruit size through coordinating the abundant cell division and expansion that occurs after anthesis [10-13]. The transition of ovary into fruit is initiated by successful pollination and fertilization [11], in which auxin plays key role in triggering the fruit-set program and initiating fruit development [14-16]. Further, several reports demonstrated the critical role played by auxin in regulating the onset and coordination of ripening processes, and subsequent fruit shelf-life [6, 11, 13, 17-19]. Recent studies have shown that auxin accelerates fruit development and ethylene production, acting at least partially by triggering the expression of several ethylene biosynthesis and response components [13, 20, 21]. Once the ripening process is initiated, it cannot be stalled and generally leads to over-ripening that in turn negatively affects fruit quality. Therefore, identifying factors that coordinate fruit ripening remains one of the biggest challenges to minimize postharvest losses. Several fruit parameters are used to specify the progression of ripening. The significant postharvest loss of fresh fruits due to excessive and rapid softening has urged considerable research into investigating the mechanisms that underlie cell-wall dynamics [19, 22, 23]. Fruit textural changes during ripening are associated with numerous modifications of the cell-wall architecture, leading to a reduction in intercellular adhesion, depolymerization and solubilization of pectins and hemicellulose, and loss of pectic galactose side chains $[24,25]$. These modifications in cell-walls involve the coordinated and interdependent action of many cell-wall modifying enzymes and proteins. Thus, investigating the developmental process and signal mechanisms involved in the regulation of cell-wall associated genes is an important area of research. Despite the wellestablished role played by ethylene in orchestrating the ripening of climacteric fruit, the role of other ethylene-independent metabolic pathways in the regulation of climacteric fruit ripening is obvious [6, 26, 27]. Indeed, several studies have accentuated the impact of auxin on regulating different aspects of fruit ripening and quality traits in many crop species [19, 28-30]. For instance, manipulation of auxin-signalling components in tomato can enhance starch accumulation, increase wall thickness of fruit epidermal cells, and reduce pectin solubilization [31-34]. However, the exact molecular mechanisms by which auxin regulates these processes are not fully understood.

It is well-established that auxin modulates plant development through transcriptional regulation of target auxin-responsive genes [3]. Therefore, the involvement of auxin in a diverse array of physiological functions should be equally mediated by a series of signalling network cascades. The discovery of the $F$-box proteins that act as auxin receptors has considerably improved our understanding of how auxin mediates cellular responses $[35,36]$. Basically, the transcriptional regulators of Aux/ IAAs and ARFs interact in homo- and heterodimers to form complexes that repress auxin-signalling. Auxin binding to the $F$-box receptor proteins promotes the formation of SCF ${ }^{\mathrm{TIR} 1 / \mathrm{AFB}}$ ubiquitin-ligase complexes that target the Aux/IAA repressors for degradation. This auxin-dependent proteolysis releases auxin response factors (ARFs) that otherwise remain trapped via their binding to Aux/IAA partners [37-40]. Loss of Aux/IAA allows ARF-mediated auxin-responsive changes in downstream gene transcription. Previous studies on tomato and Arabidopsis have identified several auxinsignalling components that act as positive or negative regulators of auxin responses, including a member of $F$ box auxin receptor (TIR1), an Aux/IAA transcription factor (IAA9), and two members of auxin-response factor, $A R F 7$ and ARF8 [14, 16, 41-43]. The alteration of these auxin-signalling components either by activation (e.g. TIR1) or suppression (e.g. IAA9, ARF7, and ARF8) causes separation of fruit initiation from pollination and fertilization.

The role of auxin during the development of plum fruits has previously been demonstrated [17-19]. It was shown that exogenous application of auxin to plum 
fruits is capable of accelerating fruit development and ripening, confirming the role of auxin during ripening. However, the molecular mechanisms underlying such responses are still lacking. In the present study, a plum auxin receptor gene, PslTIR1, was overexpressed in tomato (Solanum lycopersicum), the plant model that has extensively been used to study ripening and postharvest biology of fleshy climacteric fruits. The morphological and molecular analysis of tomato transgenic lines clearly supported the hypothesis that auxin regulates leaf morphology, fruit development, and ripening through positive regulatory mechanisms. This study not only provide better understanding to the role of auxinsignalling components during fruit ripening, but might also lead to novel strategies for effective manipulation of ripening and fruit quality traits, adding a new level of complexity to the regulation of fruit ripening.

\section{Methods}

\section{Plant materials and postharvest treatments}

Tomato plants (Solanum lycopersicum cv. Ailsa Craig) were grown under controlled conditions set as follows: 14:10 h light $/ 300 \mu \mathrm{mol} \mathrm{m}^{-2} \mathrm{~s}^{-1} ; 25: 20{ }^{\circ} \mathrm{C}$ and $80 \%$ relative humidity. For molecular analysis, leaf samples were collected from 10 week old wild-type (WT) and T3 generation transgenic tomato lines. To evaluate the effect of auxin in the accumulation of early auxin-responsive genes, $12 \mathrm{~d}$ old WT tomato seedlings were soaked in liquid MS medium with or without (mock treatment) $10 \mu \mathrm{M}$ NAA for $2 \mathrm{~h}$. For ethylene quantification, WT and transgenic tomato fruit were harvested at early immature green, immature green, mature green, breaker, orange, early red, red, red-ripe stages. Ethylene production was quantified in 5 fruit/treatment/replicate with three independent biological replicates using gas chromatography. All fruit samples were frozen in liquid- $\mathrm{N}_{2}$ and stored at $-80{ }^{\circ} \mathrm{C}$ for further analysis.

\section{Generation of transgenic tomato plants}

A high fidelity PCR system was used to amplify the fulllength sequence of PslTIR1 cDNA [18], using gene specific primers 1 and 2 (Additional file 1: Table S1). The cDNA was fused into SpeI/BstEII site of modified pCambia1304 binary vector (hygromycin resistant gene was replaced by kanamycin resistant gene) under the transcriptional control of $35 S$ promoter. The PSlTIR1 was then introduced into the WT tomato plants (Solanum lycopersicum cv. Ailsa Craig) by Agrobacterium tumefaciens-mediated transformation [32]. Transformed lines were first selected on kanamycin $\left(70 \mathrm{mg} \mathrm{L}^{-1}\right)$ and further confirmed by PCR with the genomic DNA ( $g \mathrm{DNA}$ ) extracted from leaves of 10 week old transgenic tomato lines to check the presence of TDNA insertion. To identify discrete transgenic lines, a qPCR analysis was performed to determine PslTIR1 transgene accumulation levels. Consequently, a number of independent transformation events were identified from which only two lines were selected for further analysis (L12 and L17). To determine parthenocarpic capacity, few flower buds of WT and transgenic plants were emasculated $2 \mathrm{~d}$ before anthesis to prevent self-pollination and all other flowers were removed.

\section{Nucleic acid extraction and qPCR assays}

Total RNA extraction, DNase treatment, cDNA synthesis and $\mathrm{qPCR}$ reactions were performed as described previously [44]. Gene-specific primers were designed using Primer Express (v3.0, Applied Biosystems, Carlsbad, CA, USA) (Additional file 1: Table S1). Three technical replicates from three biological replicates for each reaction were analyzed on an ABI PRISM 7900HT Sequence Detection System (Applied Biosystems). Transcript abundance was quantified using standard curves for both target genes and tomato $\beta$-actin SlAct (BT013524) as a reference gene, which were generated from serial dilutions of PCR products from corresponding cDNAs. The expression level of SlAct among different tissues and treatments used in this study was assessed using absolute qPCR. The qPCR assay was performed based on the standard curve generated from recombinant plasmids. No significant differences in SlAct expression were detected between different treatments and tissue samples. We thus conclude that SlAct could be used as a reliable internal reference gene for qPCR. Genomic DNA was extracted from tomato leaves according to the DNeasy Plant Mini Kit (Qiagen, Mississauga, ON, Canada).

\section{Post-harvest treatments and shelf-life analysis}

To evaluate the effects of auxin in fruit ripening and shelflife characteristics, WT and transgenic tomato fruit were harvested at early breaker stage ( $\sim 2 \mathrm{~d}$ after anthesis), surface sterilized, and subjected to various treatments (5 fruit/treatment/replicate; three biological replicates), including: 1-naphthalene acetic acid $(\mathrm{R}+\mathrm{N})$; NAA $(10 \mu \mathrm{M} /$ $2 \mathrm{~h})$, propylene $(\mathrm{R}+\mathrm{E}) ; \mathrm{C}_{3} \mathrm{H}_{6}\left(1000 \mu \mathrm{L} \mathrm{L}^{-1} / 24 \mathrm{~h}\right.$ at $\left.20^{\circ} \mathrm{C}\right)$, the ethylene-inhibitor 1-methylcyclopropene $(\mathrm{R}+\mathrm{M})$; 1 MCP $\left(1 \mu \mathrm{L} \mathrm{L}{ }^{-1} / 24 \mathrm{~h}\right.$ at $\left.20{ }^{\circ} \mathrm{C}\right)$; and 1 -MCP followed by dipping in NAA $(R+M+N)$. Water-dipped fruit were used as controls (R). All fruit were kept at $20{ }^{\circ} \mathrm{C}$ until reaching red stage. In case of treatments with no obvious progression in ripening such as $1-\mathrm{MCP}$ and 1-MCP/NAA treated fruit, samples were collected $\sim 20 \mathrm{~d}$ after treatment. Fruit characteristics were assessed and sampled every $5 \mathrm{~d}$ until they lost their texture and structural integrity. To determine fruit physical properties as skin puncture strength, flesh firmness and weight loss, fruit were assessed at 0, 5, 10, 15 and $20 \mathrm{~d}$ of shelf-life. Loss of fruit weight was calculated as \% of the initial fruit weight at harvest. Fruit firmness was measured in fruit with and 
without removal of skin to determine the skin punctures strength and the flesh compression mass using digital penetrometer equipped with a $3 \mathrm{~mm}$ cylinder probe (FHT200, Extech Instruments, USA). Each fruit was tested three times at equidistant points along the equatorial plane of the fruit.

\section{Statistical analysis}

The significance of differences in expression data was tested on raw data by analysis of variance adopting the General Linear Model (GLM) using SAS software. Significance between mean values was estimated by Tukey's HSD test carried out on raw data.

\section{Hierarchical clustering analysis}

Gene expression of cell-wall metabolism genes that showed significant differences between WT and PslTIR1 fruits and after exposure to different treatments that can alter auxin and ethylene signalling was grouped through a two-way hierarchical clustering. Pearson's distance and Ward's algorithm were used for data aggregation.

\section{Results and discussion}

Despite the strong sequence structure conservation among all TIR1/AFB auxin receptors that support this common auxin-signalling mechanism [45], a number of studies have shown that TIR1/AFB proteins have distinct biochemical properties and biological functions. For example, the different TIR1/AFB proteins exhibited clear divergence in their binding properties for various auxin analogs, which consequently affect their auxin-dependent ability to assemble co-receptor complex with the different Aux/IAA proteins $[46,47]$. Even in the presence of auxin, TIR1/AFBs demonstrated diverse capacities of assembling co-receptor pairs with Aux/IAAs, in which certain Aux/ IAA proteins are generally better substrates than others for a specific TIR1/AFB protein. Accordingly, Arabidopsis $A F B 3$ has been shown to have a unique role in the nitrate response of roots [48]. Also, Tomato SITIR1 and SIAFB6 have been shown to be involved in the auxin-signalling network controlling simplified leaf architecture formation [49]. Another dramatic impact that can discriminate between auxin receptors is the mechanism by how these receptors mediate auxin responses. Genetic studies indicated that the AFB4-class of auxin receptors negatively regulates the auxin-response, unlike other members of the family that act as positive regulators $[47,50,51]$. To add more level of complexity, classification of $F$-box auxin receptors gene family divided the different land plants TIR1/ AFB members into four distinguishable clades on the basis of sequence structure; TIR1, AFB2 (AFB2/AFB3), AFB4 (AFB4/AFB5), and $A F B 6[27,44]$. It is worth noting that five and three $F$-box receptor members were identified in Arabidopsis and tomato, respectively. Despite that AFB6 and AFB2 homologs are absent in Arabidopsis and tomato, respectively; the consequences of their loss remained unclear. These observations suggest that the different TIR1/AFB gene members exhibit specific and overlapping biological functions. Therefore, determining the autonomous role of the different auxin receptors in plant development is necessary to understand the fundamental contribution of each protein in auxin-dependent plant responses. Recently, three plum genes encoding proteins closely related to the TIR1-like gene family of auxin receptors (PslTIR1, PslAFB2, and PslAFB5) were characterized [18]. The results suggested that PslAFB5 is more involved in flowering and early fruit development processes with minor contribution during fruit maturation and ripening; however, both PslTIR1 and PslAFB2 proteins play important roles in mediating overall reproductive growth development. We provided a set of evidence that the three proteins are components of an SCF ubiquitin-ligase complex. They are able to assemble co-receptor complexes with different Aux/IAAs that play distinct roles in mediating auxin responses. To gain a broader insight into their potential role in plant growth and fruit development, the auxin receptor PslTIR1 was selected to evaluate the physiological and molecular consequences due to overexpression in tomato.

\section{Overexpression of PsITIR1 disturb the auxin-responsive pathway in tomato}

Several independent transgenic tomato events overexpressing plum auxin receptor, PslTIR1, were generated and tested for T-DNA insertions (Additional file 1: Figure S1). Different levels of the PslTIR1 transgene expression were detected in all lines tested (Fig. 1a); however, only two independent biological representatives (L12 and L17) were selected for further molecular and phenotypic characterization. To investigate the impact of PslTIR1-overexpression on disturbing the auxin-signalling pathway, transcript accumulation for a number of early auxin-responsive genes was assessed in WT, auxin-treated WT, and transgenic (L12 and L17) tomato seedlings (Fig. 1b). Relative to WT, transcripts for GH3.6, SAUR, and $I A A 3$ were increased markedly in PslTIR1 as well as auxintreated WT plants. GH3 genes encode IAA-amido synthetases, which converts free auxin to its conjugated form and maintains auxin homeostasis inside a cell. As in SIARF7-silenced plants [14], the up-regulation of GH3.6 indicated that its induction may compensate for excessive auxinresponse in PslTIR1-plants. Although IAA9 and ARF7 dramatically declined in all plants relative to WT, a slight difference in their accumulation pattern was detected. PslTIR1-seedlings showed more effectiveness in suppressing IAA9 mRNA ( 70 \%) than those of auxin-treated WT ( $45 \%)$. Contrary, ARF7 down-regulation was more in auxin-treated WT (80 \%) than in PslTIR1-seedlings 


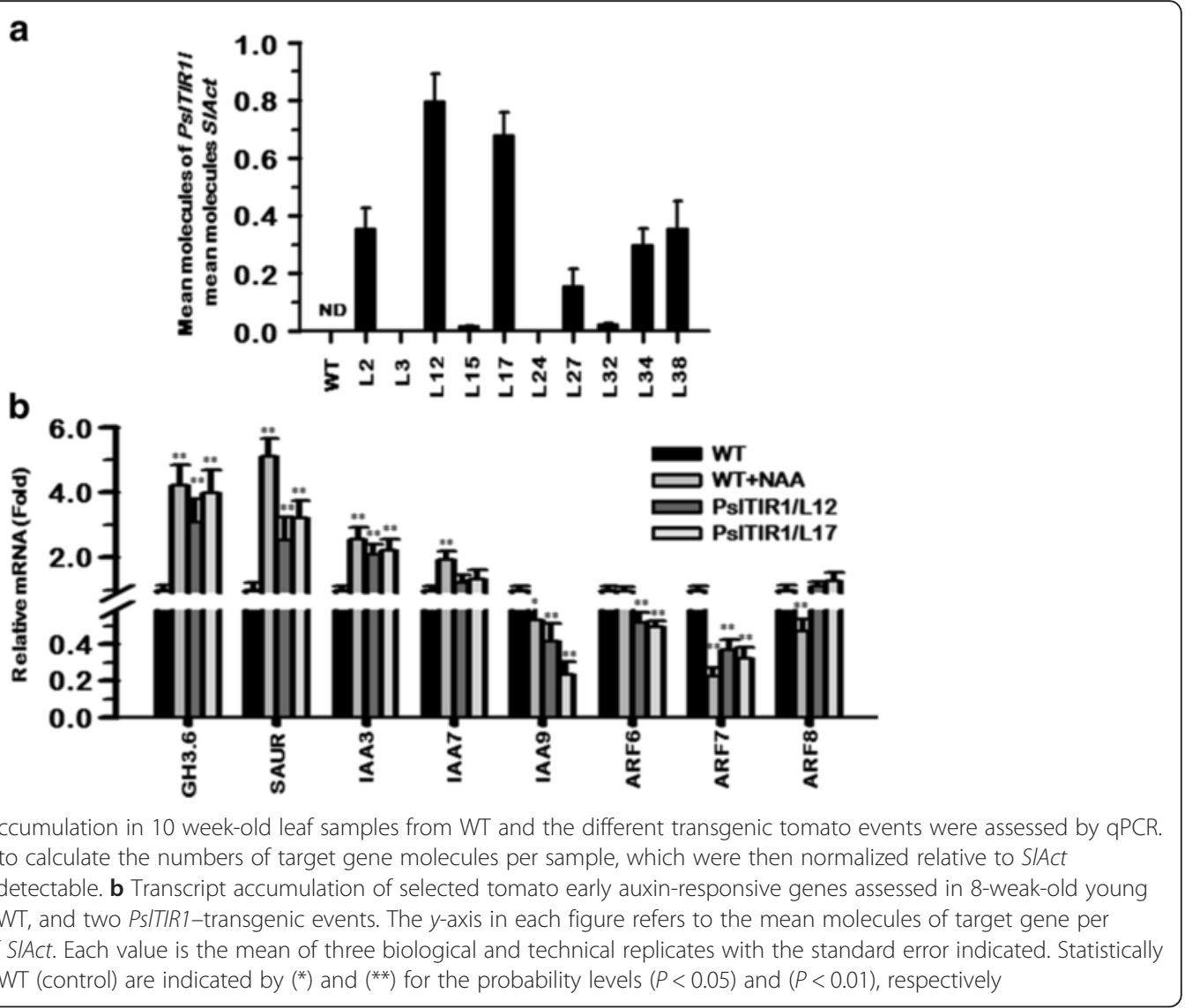

( $\sim 60 \%)$. More pronounced differences between PslTIR1-seedlings and auxin-treated WT were detected by analyzing ARF6 and ARF8 accumulation pattern. PslTIR1-seedlings exhibited significant reduction in ARF6 mRNA; however, its transcription did not respond to the auxin presence in treated WT. In contrast, no significant differences of ARF8 levels were observed in PslTIR1-seedlings, although auxin-treated WT showed considerably declined levels. Finally, the transcription of all tomato F-box/auxin receptors (TIR1, AFB4, and $A F B 6)$ was unchanged from the WT (data not shown). The differential capacities between auxin-treated WT and PslTIR1-seedlings in changing the transcription profile of the different auxin-signalling components highlighted the potential selective contribution of PSITIR1 in mediating different aspects of plant development, which can distinguish between the dynamics of auxin responses due to increasing auxin levels and that caused by activating particular auxin-signalling pathway.

PsITIR1-overexpression lines exhibit compact stature and altered leaf morphology

Overexpression of PslTIR1 led to a wide range of disturbances in general growth and development consistent with altered auxin-responsiveness. From early plant development, transgenic seedlings exhibited thicker stems and shorter internode (Fig. 2a). This altered growth continued through plant life-cycle, producing a typical dwarf phenotype with $\sim 40 \%$ reduction in the height of adult PslTIR1-plants (Fig. 2b). Interestingly, a similar phenotype was observed in transgenic tomato plants overexpressing SlTIR1 [43]. In contrast, exogenous auxin application or activating auxin-responsiveness in tomato via suppressing SIIAA9 enhanced stem elongation and produced taller plants [16, 52]. To determine the nature of this compact phenotype, we assessed the accumulation of several tomato genes and transcription factors that have been shown to contribute to plant stature with potential regulation by auxin in WT and PSlTIR1-plants, including tomato DELLA, GA2ox, GA20ox, GA3ox, TIR1, AFB5 and AFB6. However, no significant differences in their accumulation pattern were detected (data not shown), suggesting a possible autonomous role played by PslTIR1 in controlling plant stature.

Moreover, one of the most readily visible phenotype was related to leaf morphology. WT tomato leaves are unipinnately compound with a terminal leaflet and three 

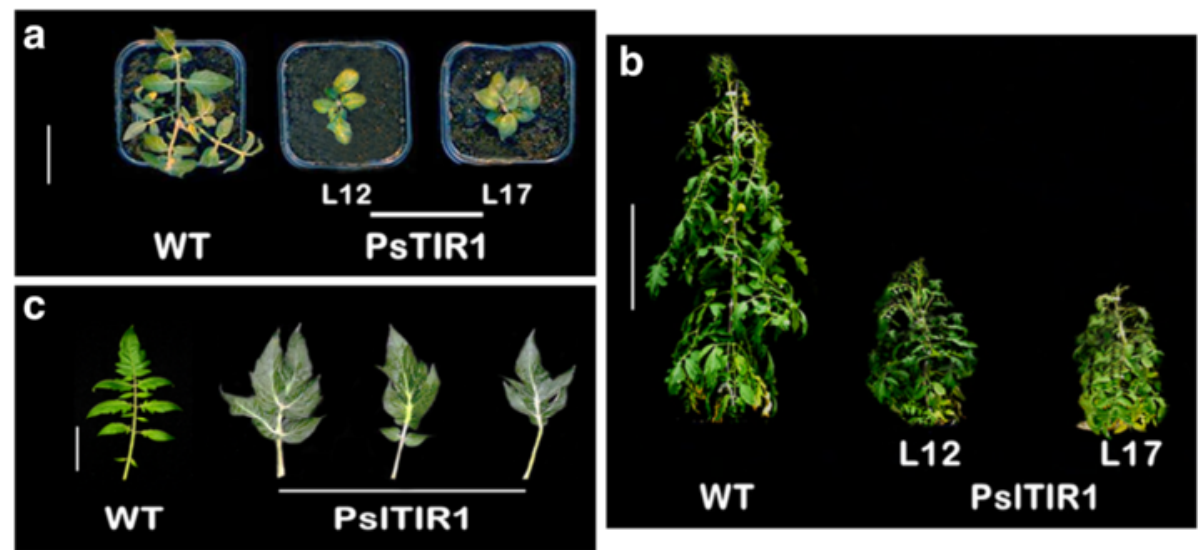

Fig. 2 Effect of PSITIR1-overexpression on vegetative growth. Phenotype representative of WT and PSITIR1-lines (L12 and L17) at two- (a) and sixteen-week old (b), respectively, (Bars $=5 \mathrm{~cm}$ and $0.3 \mathrm{~m}$, respectively). $\mathbf{c}$ Changes in leaf morphology and structure due to PSITIRT-overexpression $(\mathrm{Bar}=2.5 \mathrm{~cm})$

pairs of lobed major lateral leaflets with pinnate venation. In contrast, PslTIR1-plants displayed significant reduction in leaf compounds. They exhibited occasional lobed simple leaves architecture with fewer pairs of lateral leaflets merged with the terminal leaflet. However, the secondary small leaflets frequently seen between the major leaflets were totally absent (Fig. 2c). Auxin is the driving force of leaf growth and pinna determination $[53,54]$. Therefore, any alteration in auxin distribution or response pathways might be responsible for the changes in leaf morphology. The consistency of producing simplified leaf architecture phenotype due to TIR1overexpression from two different plant species, plum and tomato, in two different tomato genetic backgrounds, Ailsa Craig and MicroTom, suggests the definite contribution of TIR1 in patterning leaf morphogenesis and dissection ([43]; this study). So far, the tomato ENTIRE gene, also called IAA9, is the only auxin-signalling component that distinctly has been shown to mediate compound-leaf patterning via modulating auxin response $[16,49,55,56]$. Down-regulation of IAA9 in tomato reduced the complexity of leaf morphology, similar to that of PslTIR1-leaves. Several lines of evidence, including i) the capacity of PslTIR1 to form co-receptor complex with tomato IAA9 [18], ii) the dramatic suppression of IAA9 mRNA in PslTIR1-seedlings, iii) the analogous phenotype of PslTIR1- and SlTIR1-leaves morphology with those of auxin-treated tomato WT plants as well as tomato entire and IAA9 mutants [16, 43, 56] confirmed that TIR1 proteins might regulate the auxin-dependent leaf simplification via targeting the auxin repressor IAA9, leading to destabilization of IAA9::ARF inhibitory complex.
PsITIR1-overexpression causes alteration in reproductive growth behavior

WT tomato exhibits a typical coordinated fruit-set and development following pollination and fertilization [11]. PSITIR1-overexpression resulted in dramatic changes in overall reproductive growth, including flower and fruit. All transgenic plants exhibited significant reduction in the emergence of flower buds and fertility. WT produced an average of $29 \pm 5$ flowers/plant; however, PslTIR1-plants produced only $5 \pm 2$ flower/plant. PslTIR1-plants did not differ from the WT in terms of flowering time, flower size, and overall fruit development. Nonetheless, visible changes were observed in flower structure such as protruding stigma (protrudes well above the staminal cone), limiting self-pollination (Fig. 3a). The structure of open immature fused staminal cone in older flowers supported the impact of stimulated ovary growth in producing this phenotype. Our results suggested that PslTIR1-overexpression caused precocious fruit-set prior to anthesis independent of pollination and fertilization, which triggered the parthenocarpic fruit development. Fruit-set did not occur in emasculated WT flowers and the unfertilized flowers abscised within 3-4 $\mathrm{d}$. Emasculated PslTIR1-flowers, by contrast, remained attached to the plant and developed into seedless fruit (Fig. 3b, c), which confirmed parthenocarpy. Despite this parthenocarpic character, PslTIR1-fruit were similar in appearance to WT in terms of size and skin color.

These results clearly indicated that PslTIR1-overexpression caused considerable disturbance in several auxin-responsive genes, resulting in alteration on typical fruit-set program and strong tendency to develop parthenocarpic fruit. In tomato, parthenocarpy fruit-set can be induced by auxin application or by modifying auxin- 
a
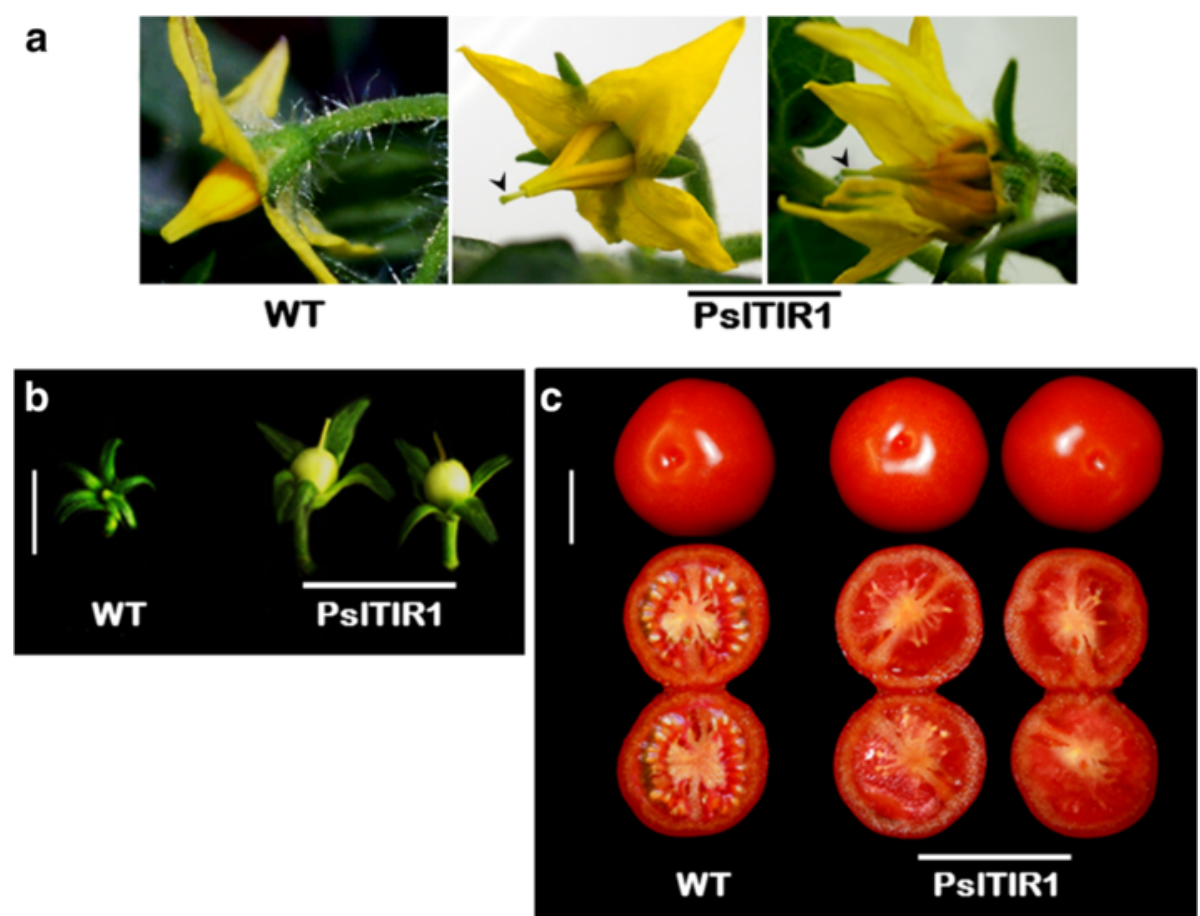

Fig. 3 Close-up views of WT and PSITIR1 a flowers at anthesis; the arrows indicate the protruding stigma. b Parthenocarpic fruit-set at early immature stage after emasculation; the stamen cones were removed when the flowers had not yet opened, but are ready to turn yellow $(B a r=50 \mathrm{~mm})$. c Adult mature fruit from WT after fertilization and parthenocarpic Ps/TIRT-fruit $(B a r=6 \mathrm{~cm})$

signalling [14, 16, 41, 42, 57]. However, several lines of evidence suggest that this phenotype is mainly due pleiotropic effect of IAA9 suppression caused by PslTIR1-overexpression. Compared with WT, the basal transcript levels of IAA9 and ARF7 were decreased in PslTIR1-lines, which agrees with previous studies reported the involvement of IAA9- and ARF7suppression in parthenocarpic fruit-set [14, 16, 43]. Apparently the disturbance in the gene network involved in fruit-set either by suppression (e.g. IAA9 and ARF7) or activation (e.g. TIR1) led finally to produce similar changes in fruit-set process. Moreover, the abundance of auxin-induced GH3.6, SAUR, and IAA3 transcripts in PslTIR1-lines is consistent with their accumulation profile in auxin-hypersensitive tomato mutants $[14,16,43,58]$. Thus, it is possible to speculate that PsITIR1 positively regulate auxin-responses and fruitset via mediating the degradation of Aux/IAA proteins, particularly IAA9.

\section{Effect of PsITIR1-overexpression in fruit ripening}

In climacteric fruits, auxin can enhance ripening and ethylene production, acting at least partially by triggering the transcription of several ethylene biosynthesis and signalling component elements $[6,13,32,59]$. To examine the contribution of PSITIR1-overexpression, we monitored the ethylene production from early immature green until red-ripe stages in WT and transgenic fruits. All fruits exhibited progressive ethylene production during ripening with no significant differences between WT and transgenic fruits (Fig. 4a). To confirm this, the accumulation profile of a set of genes that are actively involved in ethylene production and fruit ripening was assessed in red WT and transgenic fruit with or without auxin treatment, using qPCR (Fig. 4b, Additional file 1: Table S1). Analysis of expression data indicated that the accumulation profile of the different ethylene- and ripening-related transcripts in PSITIR1-fruit remained identical to that in the WT and did not visibly respond to auxin treatment, excluding those of ACS4, ACO5 and ERF1. The accumulation profile of ACS4 indicated that its transcription was triggered by auxin, but PSITIR1 is not involved in this stimulatory effect. Although ACO5 is dramatically increased in PslTIR1-fruit, its response to auxin treatment suggested the auxin-independent accumulation pattern. Interestingly, considerable high levels of ACO5 were found to be associated with tomato parthenocarpic fruit development [60]. Thus, the accumulation of ACO5 in PslTIR1-fruit seemed to be parthenocarpic-dependent rather than auxin-dependent. ERF1 showed a typical auxin-dependence accumulation in terms of response to auxin application and 


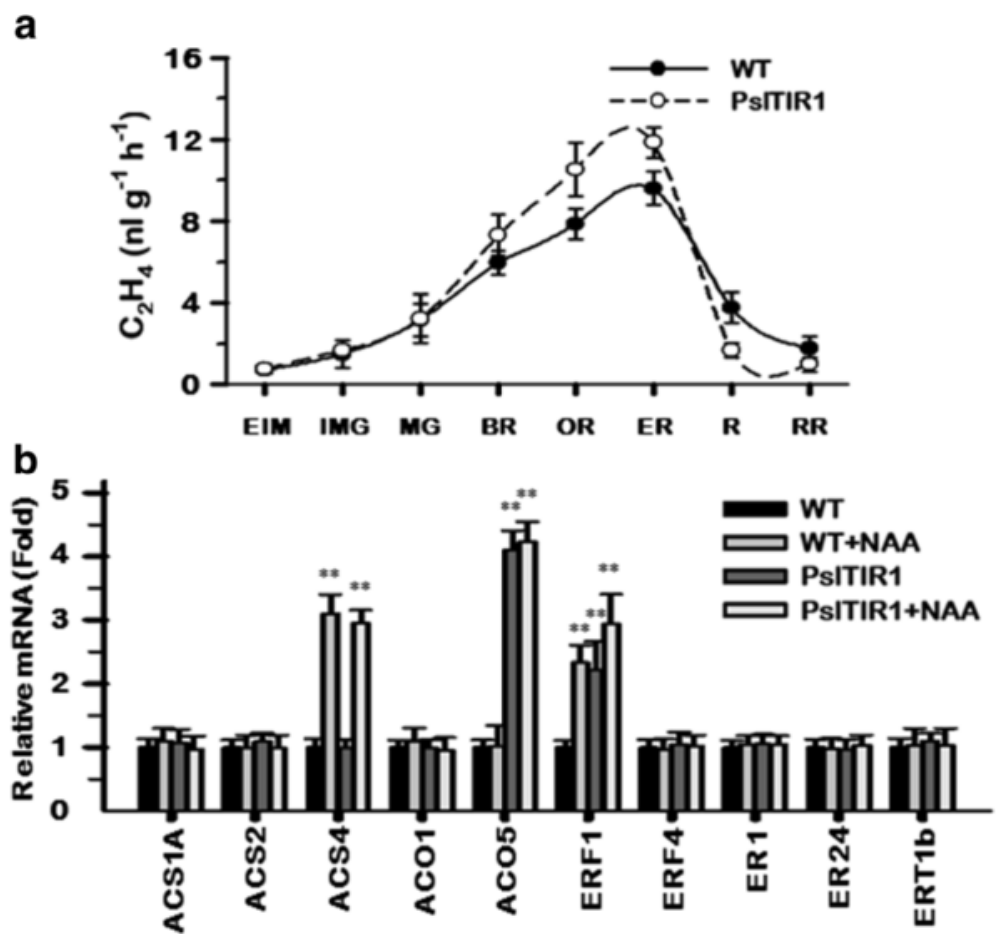

Fig. 4 a Changes in ethylene production during WT and PSITIR1-fruit development; early-immature green (EIM), immature green (IMG), mature green $(M G)$, breaker (BR), orange (OR), early red (ER), red (R), red-ripe (RR). b Transcript accumulation of selected tomato genes involved in defining ethylene production levels and fruit ripening was assessed in red WT and PSITRT-fruit treated or not with auxin. The $y$-axis in (a) represents the changes in ethylene levels $\left(\mathrm{nl} \mathrm{g} \mathrm{g}^{-1} \mathrm{~h}^{-1}\right)$. Statistically significant differences from WT (control) are indicated by $\left.{ }^{* *}\right)$ for the probability levels $(P<0.01)$. Other details as in Fig. 1

PslTIR1-dependent regulation. These results suggested that PSITIR1-overexpression is not involved in the crosstalk regulatory mechanism between ethylene and auxin signalling.

Effect of PsITIR1-overexpression in fruit shelf-life trait Ethylene and its biosynthetic genes are involved in the regulation of fruit softening and maintenance of shelflife in several fleshy fruits [61-63]. Our results suggested the minor contribution of PSITIR1 in mediating autocatalytic ethylene production and in coordinating tomato fruit ripening. This prompted us to assess the postharvest behavior of PslTIR1-fruit to determine their shelflife capacity. Texture of fleshy fruit not only affects consumer preference, but also has a significant impact on shelf-life and storability. WT and transgenic fruits were harvested at early breaker stage and stored at room temperature until they reached complete deterioration ( $20 \mathrm{~d}$ after storage). To confirm any potential role of auxin, shelf-life characteristics were also assessed in WT fruit treated with auxin. PSlTIR1 and auxin-treated WT fruits broke down faster than WT with much more and rapid deterioration in auxin-treated WT fruit (Fig. 5a).

The shelf-life reduction of PslTIR1-fruit had driven us to assess several shelf-life parameters to better evaluate the impact of auxin. The shelf-life was measured mainly by weight loss, penetration strength, and firmness during storage of fruits. Initially, no significant changes in weight loss were observed. However, as ripening proceeded, the weight loss significantly increased in PslTIR1-fruit relative to control (Fig. 5b), resulting in $70 \% \pm 5.3$ loss of weight by the end of storage period; while WT fruit exhibited only $54 \% \pm 4.7$ weight loss. Interestingly, treatment of WT with auxin increased the rate of weight loss, even higher than PslTIR1-fruit (81 \% \pm 6.6 weight loss). During storage, fruit firmness data represented by skin mechanical strength and flesh compression, showed that control WT fruit were substantially firmer than that of PSITIR1 and auxin-treated tomatoes (Fig. 5c, d). By the end of storage duration, PslTIR1 and auxin-treated fruits were $47 \%$ and $61 \%$ less in penetration mass, and 59 and $72 \%$ less in flesh firmness than control, respectively. Comparing with PslTIR1-fruit, the stronger effect of auxin treatment in fruit shelf-life characteristics suggested that PslTIR1 is not the only auxin-related protein involved in mediating fruit shelf-life events, particularly weight loss and firmness.

\section{Cell-wall metabolism genes differentially respond in PsITIR1-overexpressed tomato}

The alterations in shelf-life characteristics of PSlTIR1fruit prompted us to investigate whether PslTIR1- 


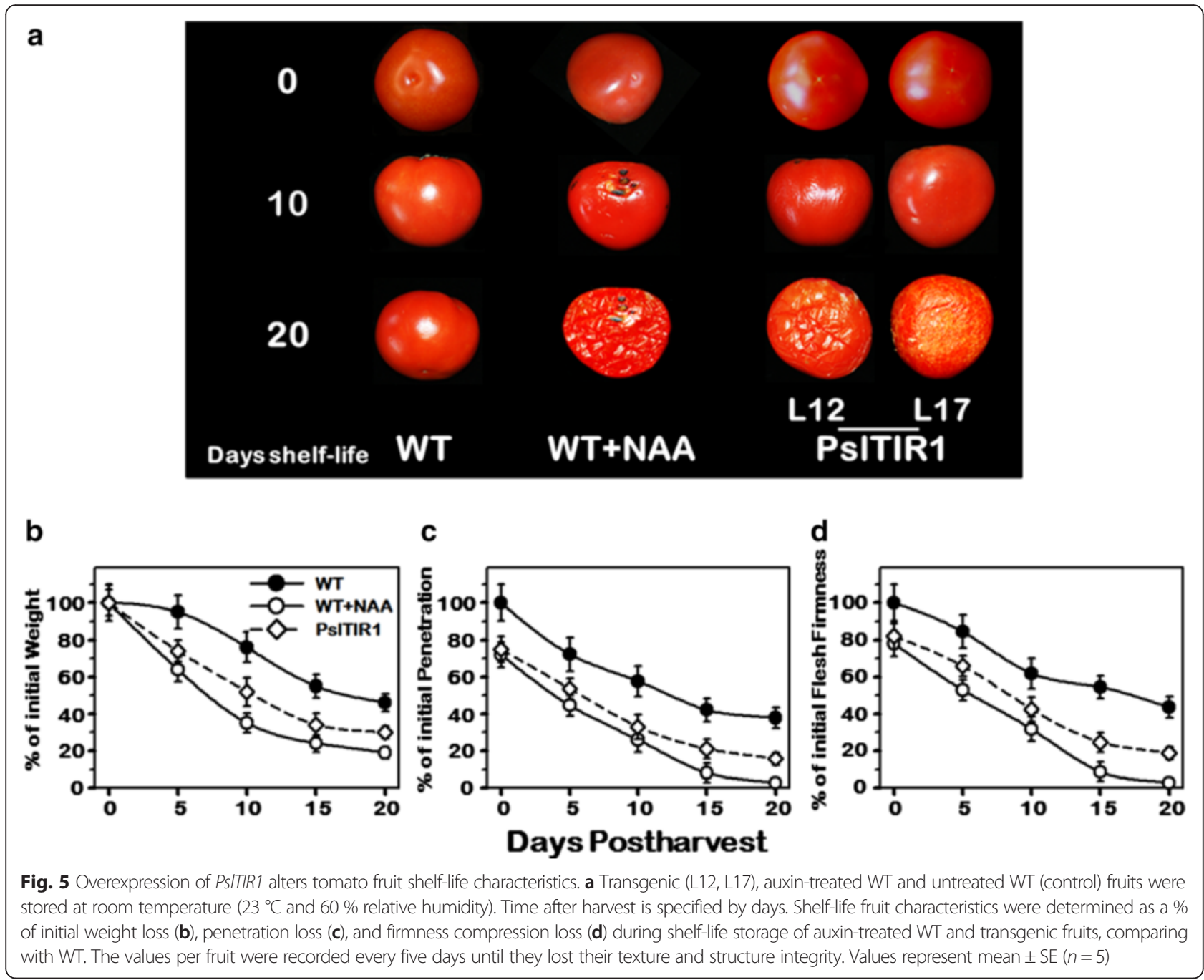

overexpression impacts genes encoding proteins involved in cell-wall degradation. To establish the regulatory mechanism(s) of fruit softening during ripening, the expression of a set of cell-wall metabolism genes that are involved in defining tomato fruit firmness (Additional file 1: Table S1) was quantified in WT and PslTIR1-fruit at harvest (early breaker stage; B) and after reaching ripening red stage. Further, the transcription of the different cell-wall metabolism genes was assessed in red WT and PslTIR1 fruits pre-exposed to several treatments that can alter auxin and ethylene signalling to determine the involvement of ethylene and auxin in fruit softening.

Among the twelve cell-wall metabolism genes tested, eight transcripts including $\beta H e x$, TomQB, EXET, EXP5, TBG4, $\beta G l u, P E$ and $C e l$ were initially higher in PslTIR1-fruit than WT at early breaker stage (Additional file 1: Figure S2). However, all the 12 transcripts dramatically increased in both WT and transgenics with the progression in fruit ripening. Analysis of expression data in red WT fruit (Ripening Control) differentiated the transcripts based on their responses to different treatments into two main groups (Fig. 6). Group-1 includes all mRNAs greatly accumulated in an ethylenedependent manner, with no visible response to any of auxin-related treatments (aMan, PME, PG, and XTH9). The second group contains all transcripts up-regulated in both auxin- and ethylene-dependent manners, including BHex, TomQB, EXET, EXP5, TBG4, $\beta G l u, P E$ and $\mathrm{Cel}$. Although their expression levels significantly declined in MCP-treated WT fruit, they considerably accumulated in PslTIR1-fruit treated with MCP or in WT and PSlTIR1-fruit treated with MCP and auxin (Additional file 1: Figure S2).

Cell-wall metabolism during ripening is an important aspect and has been explored extensively. Both ethylenedependent and -independent softening pathways coexist to coordinate climacteric fruit ripening process $[6,26]$. 


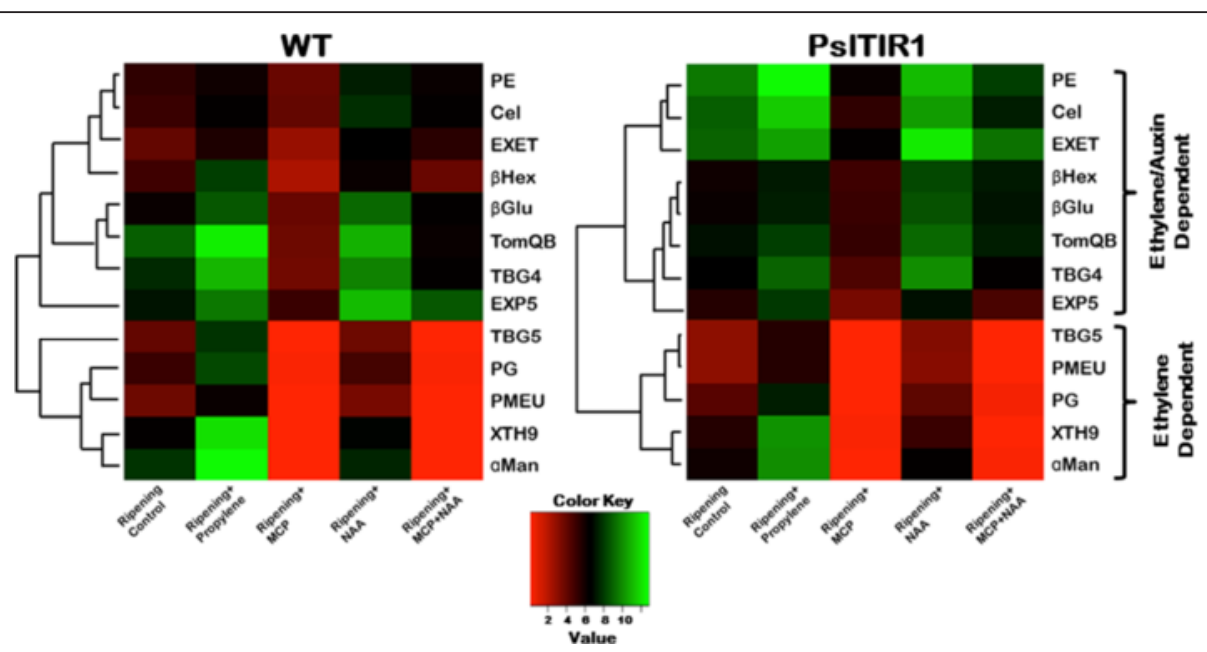

Fig. 6 Hierarchical clustering analysis of the transcript levels of cell-wall metabolism genes. The clustering analysis was performed on genes differentially expressed between WT and PSITIR1 fruits at ripening and in ripen fruits pre-exposed to different treatments that alter ethylene and auxin response. Green boxes indicate higher levels of expression, and red boxes indicate lower expression levels compared with the WT (Ripening Control). The color brightness is directly proportional to the expression ratio, according to the color scale at the bottom of the figure

Given its almost ubiquitous importance, it was not surprising that auxin plays a prominent role in coordinating different aspects of fruit ripening [1]. The impact of auxin in mediating fruit firmness by regulating the fine pectin structure and tissue architecture has been previously acknowledged [31-33]. Moreover, downregulation of tomato APETALA2a gene suggested that some of the ethylene-mediated responses are performed through auxin action, at least in part, during ripening [64].

\section{Conclusions}

Plant hormones are long known to be tightly associated with fruit development and fruit ripening. Although, ethylene is considered a major player in coordinating the ripening-related events in climacteric fruit, emerging evidences highlighted auxin as another integral player in this dynamic mechanism. The present study provides another line of evidence through the overexpression of a plum auxin receptor, PslTIR1, in tomato. Although transgenic tomato plants showed signs of auxinhypersensitivity, which are usually connected to the overexpression of auxin positive regulators, the accelerated softening of transgenic fruits represents a novel phenotype that links auxin directly to the ripening process. In our previous study, we found that the accumulation of PSITIR1 mRNA is well correlated with high ethylene levels, high auxin content, and rapid loss of firmness in plum fruit [18]. In the present study we demonstrated that PslTIR1 protein is not involved in stimulating autocatalytic ethylene production associated with fruit ripening; however, it is more implicated in fruit softening events through controlling the transcription cell-wall disassembly related genes independent of ethylene action. Altogether, this study shows another strand in the molecular network that orchestrates the progression of ripening in climacteric fruit.

\section{Availability of supporting data}

All supporting data are included as additional files.

\section{Additional file}

Additional file 1: Table S1. The oligonucleotide primers. Figure S1. PCR with genomic DNA of potential positive PSITIR1-transgenic lines. Figure S2. Steady-state transcript levels of several tomato cell-wall disassembly genes. (DOCX $251 \mathrm{~kb}$ )

\section{Abbreviations}

1-MCP: 1-methylcyclopropene; ACO: 1-aminocyclopropane-1-carboxylate oxidase; ACS: 1-aminocyclopropane-1-carboxylate synthase; AFB: Auxin Signaling F-Box; aGal: a-galactosidase; aMan: a-mannosidase; ARF: auxin response factor; Aux/IAA: auxin-signalling repressors; Cel: endo-1,4- $\beta$ glucanase; DELLA: gibberellin-signalling repressors; ERF: ethylene response factor; EXET: Endo-xyloglucan transferase; Exp: expansin; GA20ox: gibberellin 20-oxidase; GA2ox: gibberellin 2-oxidase; GA3ox: gibberellin 3-oxidase; GH3: auxin-conjugating enzyme; NAA: 1-naphthalene acetic acid; PE: Pectinesterase; PG: polygalacturonase; PME: pectin methylesterase; SAUR: small aux-in-up RNAs; SCF: Skp/Cullin/F-box complex; TBG: $\beta$ galactosidase; TIR1: transport inhibitor response 1; TomQb: Glucan endo-1,3$\beta$-D-glucosidase; WT: wild-type; XTH: xyloglucan endotransglucosylase-

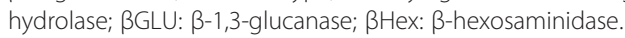

\section{Competing interests}

The authors declare that they have no competing interests.

\section{Authors' contributions}

IS and SJ conceived and designed the experiments; IS performed the molecular biology and postharvest experiments, conducted the data analysis and wrote the manuscript; SS designed and oversaw the gene expression 
experiments and participated in drafting the manuscript. BJ participated in the analyses and writing of the manuscript, WK conducted the heat-map analysis; ZL performed the genetic transformation of tomato; AS and SJ supervised the study and reviewed the manuscript. All authors read and approved the final manuscript.

\section{Acknowledgements}

We thank Glen Alm for his help during sample collection and Dr. Sadanand Dhekney, University of Wyoming, USA for critical reading of the manuscript. This work was partially supported by funds from the Ontario Tender Fruit Producers Marketing Board (OTFPMB), Early Researcher Award and Ontario Ministry of Agriculture Food and Rural Affairs (CA) to SJ.

\section{Author details}

${ }^{1}$ Department of Plant Agriculture, University of Guelph, Vineland Station, ON, Canada. ${ }^{2}$ Damanhour University, Faculty of Agriculture, Damanhour, Egypt.

${ }^{3}$ The University of Sydney, Faculty of Agriculture, Sydney, Australia.

${ }^{4}$ Chongqing University, Genetic Engineering Research Center, Bioengineering College, Chongqing, China. ${ }^{5}$ Department of Plant Agriculture, University of Guelph, Guelph, ON, Canada.

\section{Received: 7 September 2015 Accepted: 26 February 2016}

Published online: 29 February 2016

\section{References}

1. Friml J. Auxin transport-shaping the plant. Curr Opin Plant Biol. 2003;6:7-12.

2. Lüthen $H$, Claussen $M$, Böttger M. Growth: progress in auxin research. Prog Bot. 1999;60:315-40.

3. Ulmasov T, Hagen G, Guilfoyle TJ. Dimerization and DNA binding of auxin response factors. Plant J. 1999;19:309-19.

4. Quint M, Gray WM. Auxin signaling. Curr Opin Plant Biol. 2006:9:448-53.

5. Teale WD, Paponov IA, Palme K. Auxin in action: signalling, transport and the control of plant growth and development. Nat Rev Mol Cell Biol. 2006;7: 847-59.

6. Kumar R, Khurana A, Sharma AK. Role of plant hormones and their interplay in development and ripening of fleshy fruits. J Exp Bot. 2014;65:4561-75.

7. McAtee P, Karim S, Schaffer R, David K. A dynamic interplay between phytohormones is required for fruit development, maturation, and ripening. Front Plant Sci. 2013; 4:79

8. Müller A, Düchting P, Weiler EW. A multiplex GC-MS/MS technique for the sensitive and quantitative single-run analysis of acidic phytohormones and related compounds, and its application to Arabidopsis thaliana. Planta. 2002; 216:44-56.

9. Coen ES, Meyerowitz EM. The war of the whorls: genetic interactions controlling flower development. Nature. 1991;353:31-7.

10. Devoghalaere F, Doucen T, Guitton B, Keeling J, Payne W, Ling TJ, Ross JJ, Hallett IC, Gunaseelan K, Dayatilake GA. A genomics approach to understanding the role of auxin in apple (Malus $x$ domestica) fruit size control. BMC Plant Biol. 2012;12:7.

11. Gillaspy G, Ben-David H, Gruissem W. Fruits: a developmental perspective. Plant Cell. 1993:5:1439.

12. Mapelli S, Frova C, Torti G, Soressi GP. Relationship between set, development and activities of growth regulators in tomato fruits. Plant Cell Physiol. 1978;19:1281-8.

13. Trainotti $\mathrm{L}$, Tadiello A, Casadoro G. The involvement of auxin in the ripening of climacteric fruits comes of age: the hormone plays a role of its own and has an intense interplay with ethylene in ripening peaches. J Exp Bot. 2007; 58:3299-308.

14. de Jong M, Wolters-Arts $M$, Feron R, Mariani C, Vriezen WH. The Solanum lycopersicum auxin response factor 7 (SIARF7) regulates auxin signaling during tomato fruit set and development. Plant J. 2009;57:160-70.

15. De Jong $M$, Mariani $C$, Vriezen $W H$. The role of auxin and gibberellin in tomato fruit set. J Exp Bot. 2009;60:1523-32. doi:10.1093/jxb/erp094.

16. Wang H, Jones B, Li Z, Frasse P, Delalande C, Regad F, Chaabouni S, Latche A, Pech J-C, Bouzayen M. The tomato Aux/IAA transcription factor IAA9 is involved in fruit development and leaf morphogenesis. Plant Cell. 2005;17: 2676-92.

17. El-Sharkawy I, Mila I, Bouzayen M, Jayasankar S. Regulation of two germinlike protein genes during plum fruit development. J Exp Bot. 2010;61:1761-70.
18. El-Sharkawy I, Sherif SM, Jones B, Mila I, Kumar PP, Bouzayen M, Jayasankar S. TIR1-like auxin-receptors are involved in the regulation of plum fruit development. J Exp Bot. 2014;65:5205-15.

19. El-Sharkawy I, Sherif S, Qubbaj T, Sullivan AJ, Jayasankara S. Stimulated auxin levels enhance plum fruit ripening, but limit shelf-life characteristics. Postharvest Biol Tec. 2016;112:215-23.

20. El-Sharkawy I, Kim WS, Jayasankar S, Svircev AM, Brown DCW. Differential regulation of four members of ACC synthase gene family in plum. J Exp Bot 2008:59:2009-27.

21. El-Sharkawy I, Sherif S, Mila I, Bouzayen M, Jayasankar S. Molecular characterization of seven genes encoding ethylene-responsive transcriptional factors during plum fruit development and ripening. J Exp Bot. 2009;60:907-22.

22. Brummell DA, Harpster MH. Cell wall metabolism in fruit softening and quality and its manipulation in transgenic plants. Plant Mol Biol. 2001:47:311-40

23. Li X, Xu C, Korban SS, Chen K. Regulatory mechanisms of textural changes in ripening fruits. Crit Rev Plant Sci. 2010;29:222-43.

24. Fry SC. Primary cell wall metabolism: tracking the careers of wall polymers in living plant cells. New Phytol. 2004;161:641-75.

25. Rose JKC, Catalá C, Gonzalez-Carranza C, Zinnia H, Roberts J. Plant cell wall disassembly. In: The Plant Cell Wall. Oxford: Blackwell Publishing; 2003. p. 264-324.

26. Pech J-C, Bouzayen M, Latché A. Climacteric fruit ripening: ethylenedependent and independent regulation of ripening pathways in melon fruit. Plant Sci. 2008;175:114-20.

27. Pech J-C, Purgatto E, Bouzayen M, Latché A. Ethylene and fruit ripening. Annu Plant Rev Plant Horm Ethyl. 2012;44:275-304.

28. Abel S, Theologis A. Early genes and auxin action. Plant Physiol. 1996;111:9.

29. Cohen JD. In vitro tomato fruit cultures demonstrate a role for indole-3acetic acid in regulating fruit ripening. J Am Soc Hortic Sci. 1996;121:520-4.

30. Vendrell M. Dual effect of 2,4-D on ethylene production and ripening of tomato fruit tissue. Physiol Plant. 1985;64:559-63.

31. Guillon F, Philippe S, Bouchet B, Devaux M-F, Frasse P, Jones B, Bouzayen M, Lahaye M. Down-regulation of an auxin response factor in the tomato induces modification of fine pectin structure and tissue architecture. J Exp Bot. 2008:59:273-88.

32. Jones B, Frasse P, Olmos E, Zegzouti H, Li ZG, Latché A, Pech JC, Bouzayen M. Down-regulation of DR12, an auxin-response-factor homolog, in the tomato results in a pleiotropic phenotype including dark green and blotchy ripening fruit. Plant J. 2002;32:603-13.

33. Sagar M, Chervin C, Mila I, Hao Y, Roustan J-P, Benichou M, Gibon Y, Biais B, Maury P, Latché A. SIARF4, an auxin response factor involved in the control of sugar metabolism during tomato fruit development. Plant Physiol. 2013; 161:1362-74.

34. Sagar M, Chervin C, Roustan J-P, Bouzayen M, Zouine M. Under-expression of the Auxin Response Factor SI-ARF4 improves post-harvest behavior of tomato fruits. Plant Signal Behav. 2014;8:e25647.

35. Dharmasiri N, Dharmasiri S, Weijers D, Lechner E, Yamada M, Hobbie L, Ehrismann JS, Jürgens $\mathrm{G}$, Estelle M. Plant development is regulated by a family of auxin receptor F box proteins. Dev Cell. 2005;9:109-19.

36. Kepinski S, Leyser $\mathrm{O}$. The Arabidopsis F-box protein TIR1 is an auxin receptor Nature. 2005:435:446-51

37. Dharmasiri N, Estelle M. Auxin signaling and regulated protein degradation. Trends Plant Sci. 2004;9:302-8.

38. Leyser O. Dynamic integration of auxin transport and signalling. Curr Biol. 2006;16:R424-33.

39. Dharmasiri N, Dharmasiri S, Jones AM, Estelle M. Auxin action in a cell-free system. Curr Biol. 2003;13:1418-22.

40. Gray WM, Kepinski S, Rouse D, Leyser O, Estelle M. Auxin regulates SCF ${ }^{\text {TIR1 }}$ dependent degradation of AUX/IAA proteins. Nature. 2001;414:271-6.

41. Goetz M, Vivian-Smith A, Johnson SD, Koltunow AM. AUXIN RESPONSE FACTOR8 is a negative regulator of fruit initiation in Arabidopsis. Plant Cell. 2006;18:1873-86.

42. Goetz M, Hooper LC, Johnson SD, Rodrigues JCM, Vivian-Smith A, Koltunow AM. Expression of aberrant forms of AUXIN RESPONSE FACTOR8 stimulates parthenocarpy in Arabidopsis and tomato. Plant Physiol. 2007:145:351-66.

43. Ren Z, Li Z, Miao Q, Yang Y, Deng W, Hao Y. The auxin receptor homologue in Solanum lycopersicum stimulates tomato fruit set and leaf morphogenesis. J Exp Bot. 2011;62:2815-26.

44. El-Sharkawy I, El Kayal W, Prasath D, Fernandez H, Bouzayen M, Svircev AM, Jayasankar S. Identification and genetic characterization of a gibberellin 2- 
oxidase gene that controls tree stature and reproductive growth in plum. J Exp Bot. 2012;63:1225-39.

45. Tan X, Calderon-Villalobos LIA, Sharon M, Zheng C, Robinson CV, Estelle M, Zheng N. Mechanism of auxin perception by the TIR1 ubiquitin ligase. Nature. 2007:446:640-5.

46. Calderón-Villalobos LI, Lee S, De Oliveira C, Ivetac A, Brandt W, Armitage L, Sheard LB, Tan X, Parry G, Mao H. A combinatorial TIR1/AFB-AuX/AA coreceptor system for differential sensing of auxin. Nat Chem Biol. 2012;8:477-85.

47. Parry $\mathrm{G}$, Calderon-Villalobos LI, Prigge M, Peret B, Dharmasiri S, Itoh H, Lechner E, Gray WM, Bennett M, Estelle M. Complex regulation of the TIR1/ AFB family of auxin receptors. Proc Natl Acad Sci U S A. 2009;106:22540-5.

48. Vidal EA, Araus V, Lu C, Parry G, Green PJ, Coruzzi GM, Gutiérrez RA. Nitrateresponsive miR393/AFB3 regulatory module controls root system architecture in Arabidopsis thaliana. Proc Natl Acad Sci. 2010;107:4477-82.

49. Ben-Gera H, Shwartz I, Shao M-R, Shani E, Estelle M, Ori N. ENTIRE and GOBLET promote leaflet development in tomato by modulating auxin response. Plant J. 2012;70:903-15.

50. Dharmasiri N, Dharmasiri S, Estelle M. The F-box protein TIR1 is an auxin receptor. Nature. 2005;435:441-5.

51. Greenham K, Santner A, Castillejo C, Mooney S, Sairanen I, Ljung K, Estelle $M$. The AFB4 auxin receptor is a negative regulator of auxin signaling in seedlings. Curr Biol. 2011;21:520-5.

52. Yang T, Davies PJ, Reid JB. Genetic dissection of the relative roles of auxin and gibberellin in the regulation of stem elongation in intact light-grown peas. Plant Physiol. 1996;110:1029-34.

53. Barkoulas M, Hay A, Kougioumoutzi E, Tsiantis M. A developmental framework for dissected leaf formation in the Arabidopsis relative Cardamine hirsuta. Nat Genet. 2008;40:1136-41.

54. DeMason DA, Chawla R. Roles for auxin during morphogenesis of the compound leaves of pea (Pisum sativum). Planta. 2004;218:435-48.

55. Berger Y, Harpaz-Saad S, Brand A, Melnik H, Sirding N, Alvarez JP, Zinder M, Samach A, Eshed Y, Ori N. The NAC-domain transcription factor GOBLET specifies leaflet boundaries in compound tomato leaves. Development. 2009;136:823-32.

56. Zhang J, Chen R, Xiao J, Qian C, Wang T, Li H, Ouyang B, Ye Z. A single-base deletion mutation in SIIAA9 gene causes tomato (Solanum lycopersicum) entire mutant. J Plant Res. 2007;120:671-8.

57. Martinelli F, Uratsu SL, Reagan RL, Chen Y, Tricoli D, Fiehn O, Rocke DM, Gasser CS, Dandekar AM: Gene regulation in parthenocarpic tomato fruit. J Exp Bot. 2009;60(13):3873-90.

58. Chaabouni S, Jones B, Delalande C, Wang H, Li Z, Mila I, Frasse P, Latche A Pech J-C, Bouzayen M. SI-IAA3, a tomato Aux/IAA at the crossroads of auxin and ethylene signalling involved in differential growth. J Exp Bot. 2009;60: 1349-62.

59. Lin Z, Zhong S, Grierson D. Recent advances in ethylene research. J Exp Bot. 2009:60:3311-36.

60. Pascual L, Blanca JM, Cañizares J, Nuez F. Transcriptomic analysis of tomato carpel development reveals alterations in ethylene and gibberellin synthesis during pat3/pat4 parthenocarpic fruit set. BMC Plant Biol. 2009;9:67.

61. Lopez-Gomez R, Cabrera-Ponce JL, Saucedo-Arias LJ, Carreto-Montoya L, Villanueva-Arce R, Diaz-Perez JC, Gomez-Lim MA, Herrera-Estrella L. Ripening in papaya fruit is altered by ACC oxidase cosuppression. Transgenic Res. 2009;18:89-97.

62. Nishiyama K, Guis M, Rose JKC, Kubo Y, Bennett KA, Wangjin L, Kato K, Ushijima K, Nakano R, Inaba A, Bouzayen M, Latché A, Pech JC, Bennett AB. Ethylene regulation of fruit softening and cell wall disassembly in Charentais melon. J Exp Bot. 2007:58:1281-90.

63. Xiong AS, Yao QH, Peng RH, Li X, Han PL, Fan HQ. Different effects on ACC oxidase gene silencing triggered by RNA interference in transgenic tomato. Plant Cell Rep. 2005;23:639-46.

64. Karlova R, Rosin FM, Busscher-Lange J, Parapunova V, Do PT, Fernie AR, Fraser PD, Baxter C, Angenent GC, de Maagd RA. Transcriptome and metabolite profiling show that APETALA2a is a major regulator of tomato fruit ripening. Plant Cell. 2011;23:923-41.

\section{Submit your next manuscript to BioMed Central and we will help you at every step:}

- We accept pre-submission inquiries

- Our selector tool helps you to find the most relevant journal

- We provide round the clock customer support

- Convenient online submission

- Thorough peer review

- Inclusion in PubMed and all major indexing services

- Maximum visibility for your research

Submit your manuscript at www.biomedcentral.com/submit
Biomed Central 